Bull. Korean Math. Soc. 51 (2014), No. 1, pp. 55-66

http://dx.doi.org/10.4134/BKMS.2014.51.1.055

\title{
EXTINCTION AND NON-EXTINCTION OF SOLUTIONS TO A FAST DIFFUSIVE $p$-LAPLACE EQUATION WITH A NONLOCAL SOURCE
}

\author{
Yuzhu Han, Wenjie GaO, and Haixia Li
}

\begin{abstract}
In this paper, the authors establish the conditions for the extinction of solutions, in finite time, of the fast diffusive $p$-Laplace equation $u_{t}=\operatorname{div}\left(|\nabla u|^{p-2} \nabla u\right)+a \int_{\Omega} u^{q}(y, t) d y, 1<p<2$, in a bounded domain $\Omega \subset R^{N}$ with $N \geq 1$. More precisely, it is shown that if $q>p-1$, any solution vanishes in finite time when the initial datum or the coefficient $a$ or the Lebesgue measure of the domain is small, and if $0<q<p-1$, there exists a solution which is positive in $\Omega$ for all $t>0$. For the critical case $q=p-1$, whether the solutions vanish in finite time or not depends crucially on the value of $a \mu$, where $\mu=\int_{\Omega} \phi^{p-1}(x) \mathrm{d} x$ and $\phi$ is the unique positive solution of the elliptic problem $-\operatorname{div}\left(|\nabla \phi|^{p-2} \nabla \phi\right)=1, x \in \Omega$; $\phi(x)=0, x \in \partial \Omega$. This is a main difference between equations with local and nonlocal sources.
\end{abstract}

\section{Introduction}

In this short paper, we investigate the following fast diffusive $p$-Laplace equation with a nonlocal source

$$
\begin{cases}u_{t}=\operatorname{div}\left(|\nabla u|^{p-2} \nabla u\right)+a \int_{\Omega} u^{q}(y, t) d y, & x \in \Omega, t>0, \\ u(x, t)=0, & x \in \partial \Omega, t>0, \\ u(x, 0)=u_{0}(x), & x \in \Omega,\end{cases}
$$

with $1<p<2$ and $a, q>0$, where $\Omega$ is a bounded domain in $R^{N}(N \geq 1)$ with smooth boundary $\partial \Omega$, and $u_{0}(x) \in L^{\infty}(\Omega) \cap W_{0}^{1, p}(\Omega)$ is a nonnegative function. Problems of this form appear, for example, in the theory of nonNewtonian fluids and the nonlocal growth terms present a more realistic model of a population (see $[6,18]$ ). What we are interested in here is the extinction in finite time of the nonnegative solutions of (1.1), i.e., there exists a finite time

Received May 16, 2012; Revised September 12, 2012.

2010 Mathematics Subject Classification. 35B33, 35K57.

Key words and phrases. p-Laplace equation, nonlocal source, extinction.

Supported by NSFC (11271154), by Key Lab of Symbolic Computation and Knowledge Engineering of Ministry of Education and by the 985 program of Jilin University. The first author is also supported by Graduate Innovation Fund of Jilin University (20121050). 
$T>0$ such that the solution is nontrivial for $0<t<T$, but $u(x, t) \equiv 0$ for almost every $(x, t) \in \Omega \times[T, \infty)$. In this case, $T$ is called the extinction time.

Extinction of solutions is one of the most important features of many evolutionary equations that has been the subject of intensive study during the past few decades. Extinction via fast diffusion was first proved by E. Sabinina [22], and since then, there has been increasing interest in this direction (see $[4,14,16])$. Extinction as well as localization and finite propagation properties of solutions to the following semilinear problem

$$
\begin{cases}u_{t}=\Delta u-u^{q}, & x \in \Omega, t>0, \\ u(x, t)=0, & x \in \partial \Omega, t>0, \\ u(x, 0)=u_{0}(x), & x \in \Omega,\end{cases}
$$

was investigated by Kalashnikov in the 1970s and one may refer to his fundamental survey [17]. In 1994, a more complete extinction conclusion of Problem (1.2) was given in [12]: A nontrivial solution of (1.2) vanishes in finite time if and only if $0<q<1$, which means that strong absorption will cause extinction to occur in finite time.

In [12], Gu also gave a simple statement of the necessary and sufficient conditions of extinction of the solution to the following problem:

$$
\begin{cases}u_{t}=\operatorname{div}\left(|\nabla u|^{p-2} \nabla u\right)-a u^{q}, & x \in \Omega, t>0, \\ u(x, t)=0, & x \in \partial \Omega, t>0, \\ u(x, 0)=u_{0}(x), & x \in \Omega,\end{cases}
$$

with $a, q>0$. He proved that if $p \in(1,2)$ or $q \in(0,1)$ the solutions of the problem vanish in finite time, but if $p \geq 2$ and $q \geq 1$, there is no extinction. In the absence of absorption (i.e., $a=0$ ), Dibenedetto [2] and Yuan et al. [26] proved that the necessary and sufficient conditions for the extinction to occur is $p \in(1,2)$.

Later in [24], Yin and Jin studied the fast diffusive $p$-Laplace problem with a local source

$$
\begin{cases}u_{t}=\operatorname{div}\left(|\nabla u|^{p-2} \nabla u\right)+a u^{q}, & x \in \Omega, t>0, \\ u(x, t)=0, & x \in \partial \Omega, t>0, \\ u(x, 0)=u_{0}(x), & x \in \Omega,\end{cases}
$$

with $1<p<2, a, q>0$ and the dimension $N>2$. They proved that if $q>p-$ 1 , then any bounded and non-negative weak solution of Problem (1.4) vanishes in finite time for appropriately small initial data $u_{0}$, while Problem (1.4) admits at least one bounded non-negative and non-extinction weak solution for the case of $0<q<p-1$. As for the critical case $q=p-1$, whether the solutions vanish in finite time or not depends on the comparison between $a$ and $\lambda_{1}$, where $\lambda_{1}$ is the first eigenvalue of the following eigenvalue problem

$$
-\operatorname{div}\left(|\nabla \phi|^{p-2} \nabla \phi\right)=\lambda|\phi|^{p-2} \phi, x \in \Omega ; \phi(x)=0, x \in \partial \Omega .
$$


Extinction and non-extinction results similar to the ones in [24] were also obtained by Tian and $\mathrm{Mu}$ in [23], and some sufficient conditions in [24] for the solutions of (1.4) to vanish in finite time were weakened by Liu and $\mathrm{Wu}$ (see $[21])$.

There are some other extinction results of the solutions of degenerate or singular parabolic problems with or without absorption (reaction) terms, readers may refer to $[1,3,5,7,8,9,10,11,15,25]$ and references therein.

Generally speaking, for Problems (1.2) and (1.3), there is a cooperation between the diffusion term and the absorption term, and fast diffusion or strong absorption might cause any bounded nonnegative solution to vanish in finite time. However, in (1.4), the nonlinear term is physically called the "hot source", while in (1.2) and (1.3) the nonlinear term $-u^{q}$ is usually called the "cool source". Results in $[19,23,24]$ imply that when the diffusion is fast enough, the solutions might still vanish in finite time for small initial data in spite of the "hot sources".

To the best of our knowledge, extinction results of solutions to nonlocal parabolic problems were only found in [20] for the semi-linear case and in [13] for porous medium equation. Motivated by the works mentioned above, we shall study the extinction phenomena of the solutions to Problem (1.1) and give some sufficient conditions for the solutions to vanish in finite time. However, it will be seen from the following sections that, for the nonlocal problems under consideration, the first eigenvalue $\lambda_{1}$ no longer plays the same role as it does in $[19,21,23,24]$, which is the intrinsic difference between problems with local and nonlocal sources. More precisely, it is shown that if $q>p-1$, any solution with small initial datum or small coefficient $a$ vanishes in finite time, and if $0<q<p-1$, there exists a solution which is positive in $\Omega$ for all $t>0$. For the critical case $q=p-1$, whether the solutions vanish in finite time or not depends on the value of $a \mu$, where $\mu=\int_{\Omega} \phi^{p-1}(x) \mathrm{d} x$ and $\phi$ is the unique positive solution of the elliptic problem $-\operatorname{div}\left(|\nabla \phi|^{p-2} \nabla \phi\right)=1, x \in \Omega ; \phi(x)=0, x \in \partial \Omega$.

The rest of this paper is organized as follows. In Section 2, we introduce some definitions and notations and some other preliminaries. The proofs of the main results will be presented in Section 3 .

\section{Preliminaries}

In this section, as preliminaries, we introduce some definitions and notations. It is well known that the equation in (1.1) is singular at the points where $|\nabla u|=0$, and hence there is no classical solution in general. To state the definition of the weak solutions, we first introduce some notations which will be used throughout this paper:

$$
\begin{aligned}
& Q=\Omega \times(0, \infty), \quad Q_{T}=\Omega \times(0, T), \quad Q_{\left(t_{1}, t_{2}\right)}=\Omega \times\left(t_{1}, t_{2}\right), \\
& E=\left\{u \in L^{2 q}\left(Q_{T}\right) \cap L^{2}\left(Q_{T}\right) ; \frac{\partial u}{\partial t} \in L^{2}\left(Q_{T}\right), \nabla u \in L^{p}\left(Q_{T}\right) \text { for any } T>0\right\},
\end{aligned}
$$




$$
\begin{aligned}
& \widetilde{E}=\left\{u \in L^{2}\left(Q_{T}\right) ; \frac{\partial u}{\partial t} \in L^{2}\left(Q_{T}\right), \nabla u \in L^{p}\left(Q_{T}\right) \text { for any } T>0\right\}, \\
& E_{0}=\left\{u \in E ;\left.u\right|_{\partial \Omega}=0\right\}, \widetilde{E}_{0}=\left\{u \in \widetilde{E} ;\left.u\right|_{\partial \Omega}=0\right\} .
\end{aligned}
$$

Definition 2.1. A function $u \in E$ is called a weak sub-solution of Problem (1.1) provided that for any $T>0$ and any $0 \leq \varphi \in \widetilde{E}_{0}$ the following conditions hold:

$$
\begin{cases}u(x, 0) \leq u_{0}(x), & x \in \Omega, \\ u(x, t) \leq 0, & (x, t) \in \partial \Omega \times(0, T) \\ \iint_{Q_{T}} \frac{\partial u}{\partial t} \varphi d x d t+\iint_{Q_{T}}|\nabla u|^{p-2} \nabla u \nabla \varphi d x d t & \\ \leq a \iint_{Q_{T}}\left(\int_{\Omega} u^{q}(y, t) d y\right) \varphi(x, t) d x d t, & (x, t) \in \Omega \times(0, T)\end{cases}
$$

Replacing ' $\leq$ ' by ' $\geq$ ' in the above inequalities, the definition of a weak supersolution follows. Furthermore, if $u$ is a weak super-solution as well as a weak sub-solution, then we call it a weak solution of Problem (1.1).

The local existence of such a weak solution can be obtained by similar argument to that in [24], by using Schaefer's Fixed Point Theorem and a priori estimates. We only state the existence result for completeness.

Proposition 2.1. Assume that $0 \leq u_{0}(x) \in L^{\infty}(\Omega) \cap W_{0}^{1, p}(\Omega)$. If $q \geq 1$, then there exists a $T=T\left(u_{0}\right)>0$ such that Problem (1.1) admits a unique bounded and nonnegative weak solution in the cylinder $Q_{T}$; while if $q<1$, Problem (1.1) admits at least one nonnegative weak solution.

In order to prove the non-extinction results, the following comparison lemma is needed, of which the proof is more or less standard, and the readers may refer to [24] for its details.

Lemma 2.1. Suppose that $q \geq 1$. Let $u, v$ be a pair of weak super and subsolution of Problem (1.1), and $v$ is bounded, then $u \geq v$ a.e. in $Q_{T}$. In addition, if $q=1$, the boundedness of $v$ is unnecessary.

\section{Proofs of the main results}

In this section, we prove our main results and give some sufficient conditions for the solutions of (1.1) to vanish in finite time or not, by using the method of comparison principle and the integral estimates. As a matter of convenience, in what follows, we might as well assume that the weak solution is appropriately smooth, or else, we can consider the corresponding regularized problem and through an approximate process, the same result can also be obtained.

Theorem 3.1. Assume that $0 \leq u_{0}(x) \in L^{\infty}(\Omega) \cap W_{0}^{1, p}(\Omega)$ and $q>p-1$. Then every solution to Problem (1.1) vanishes in finite time provided that the initial datum or the coefficient a or $|\Omega|$ is suitably small. 
Proof. The proof will be divided into two steps. Multiplying the first equation of (1.1) by $u^{s}(s>0)$, and integrating the resulting equality over $\Omega$, one obtains $(3.1)$

$$
\frac{1}{s+1} \frac{d}{d t} \int_{\Omega} u^{s+1} d x+\frac{s p^{p}}{(s+p-1)^{p}} \int_{\Omega}\left|\nabla u^{\frac{s+p-1}{p}}\right|^{p} d x=a \int_{\Omega} u^{q} d x \int_{\Omega} u^{s} d x .
$$

Step 1: $q \leq 1$. This step will still be divided into two cases.

Case 1: $N=1$. Fix $s=1$ in (3.1). By using the Sobolev embedding inequality one has

$$
|\Omega|^{-\frac{1}{2}}\left(\int_{\Omega} u^{2} d x\right)^{\frac{1}{2}} \leq\|u\|_{L^{\infty}} \leq \gamma\left(\int_{\Omega}|\nabla u|^{p} d x\right)^{\frac{1}{p}},
$$

where $\gamma>0$ is the embedding constant, depending only on $p, N$ and $\Omega$. Combining the above inequality with (3.1) and applying Hölder's inequality on the right hand side of (3.1) yield

$$
\frac{1}{2} \frac{d}{d t} \int_{\Omega} u^{2} d x+\gamma^{-p}|\Omega|^{-\frac{p}{2}}\left(\int_{\Omega} u^{2} d x\right)^{\frac{p}{2}} \leq a|\Omega|^{\frac{3-q}{2}}\left(\int_{\Omega} u^{2} d x\right)^{\frac{1+q}{2}},
$$

or equivalently

$$
\frac{1}{2} \frac{d}{d t} \int_{\Omega} u^{2} d x \leq-|\Omega|^{-\frac{p}{2}}\left(\int_{\Omega} u^{2} d x\right)^{\frac{p}{2}}\left(\gamma^{-p}-a|\Omega|^{\frac{3+p-q}{2}}\left(\int_{\Omega} u^{2} d x\right)^{\frac{q+1-p}{2}}\right) .
$$

Since $q>p-1$, we deduce from the above inequality that if $u_{0}$ or $a$ or $|\Omega|$ is suitably small such that

$$
\gamma^{-p}-a|\Omega|^{\frac{3+p-q}{2}}\left(\int_{\Omega} u_{0}^{2} d x\right)^{\frac{q+1-p}{2}}>0
$$

then we arrive at

$$
\frac{1}{2} \frac{d}{d t} \int_{\Omega} u^{2} d x \leq-C_{0}\left(\int_{\Omega} u^{2} d x\right)^{\frac{p}{2}}
$$

where

$$
C_{0}=|\Omega|^{-\frac{p}{2}}\left(\gamma^{-p}-a|\Omega|^{\frac{3+p-q}{2}}\left(\int_{\Omega} u_{0}^{2} d x\right)^{\frac{q+1-p}{2}}\right) .
$$

Integrating $(3.2)$ over $(0, t)$ one obtains

$$
\left(\int_{\Omega} u^{2} d x\right)^{1-\frac{p}{2}} \leq\left(\int_{\Omega} u_{0}^{2}(x) d x\right)^{1-\frac{p}{2}}-(2-p) C_{0} t
$$

as long as the right hand side remains nonnegative. Thus we have

$$
\int_{\Omega} u^{2} d x \leq\left[\left(\int_{\Omega} u_{0}^{2}(x) d x\right)^{\frac{2-p}{2}}-(2-p) C_{0} t\right]_{+}^{\frac{2}{2-p}} .
$$

Then we see that $u$ vanishes at the finite time $T=\frac{\left(\int_{\Omega} u_{0}^{2} d x\right)^{1-\frac{p}{2}}}{C_{0}(2-p)}$. 
Case 2: $N \geq 2$. (a) For $\frac{2 N}{N+2}<p<2$, let $s=1$ in (3.1). By using again Hölder's inequality and the Sobolev embedding inequality, one has $(3.3)$

$$
\int_{\Omega} u^{2} d x \leq|\Omega|^{1-\frac{2(N-p)}{N p}}\left(\int_{\Omega} u^{\frac{N p}{N-p}} d x\right)^{\frac{2(N-p)}{N p}} \leq \gamma^{2}|\Omega|^{1-\frac{2(N-p)}{N p}}\left(\int_{\Omega}|\nabla u|^{p} d x\right)^{\frac{2}{p}} .
$$

Combining (3.3) with (3.1) and making use of Hölder's inequality on the right hand side of (3.1) give us the following inequality

$$
\frac{1}{2} \frac{d}{d t} \int_{\Omega} u^{2} d x+\gamma^{-p}|\Omega|^{\frac{N-p}{N}-\frac{p}{2}}\left(\int_{\Omega} u^{2} d x\right)^{\frac{p}{2}} \leq a|\Omega|^{\frac{3-q}{2}}\left(\int_{\Omega} u^{2} d x\right)^{\frac{1+q}{2}},
$$

or equivalently,

$$
\frac{1}{2} \frac{d}{d t} \int_{\Omega} u^{2} d x \leq-\left(\int_{\Omega} u^{2} d x\right)^{\frac{p}{2}}\left\{\gamma^{-p}|\Omega|^{\frac{N-p}{N}-\frac{p}{2}}-a|\Omega|^{\frac{3-q}{2}}\left(\int_{\Omega} u^{2} d x\right)^{\frac{1+q-p}{2}}\right\} .
$$

Since $p-1<q \leq 1$, we see from the above inequality that if $u_{0}$ or $a$ or $|\Omega|$ is suitably small such that

$$
\gamma^{-p}>a|\Omega|^{\frac{1-q+p}{2}}+\frac{p}{N}\left(\int_{\Omega} u_{0}^{2}(x) d x\right)^{\frac{1+q-p}{2}},
$$

then we have

$$
\frac{1}{2} \frac{d}{d t} \int_{\Omega} u^{2} d x \leq-C_{1}\left(\int_{\Omega} u^{2} d x\right)^{\frac{p}{2}}
$$

where

$$
C_{1}=\gamma^{-p}|\Omega|^{\frac{N-p}{N}-\frac{p}{2}}-a|\Omega|^{\frac{3-q}{2}}\left(\int_{\Omega} u_{0}^{2}(x) d x\right)^{\frac{1+q-p}{2}}>0 .
$$

Integrating (3.4) from 0 to $t$, one gets

$$
\left(\int_{\Omega} u^{2} d x\right)^{1-\frac{p}{2}} \leq\left(\int_{\Omega} u_{0}^{2}(x) d x\right)^{1-\frac{p}{2}}-(2-p) C_{1} t
$$

as long as the right hand side is nonnegative. Thus we have

$$
\int_{\Omega} u^{2} d x \leq\left[\left(\int_{\Omega} u_{0}^{2}(x) d x\right)^{\frac{2-p}{2}}-(2-p) C_{1} t\right]_{+}^{\frac{2}{2-p}},
$$

which implies that $u$ vanishes at the finite time $T=\frac{\left(\int_{\Omega} u_{0}^{2} d x\right)^{1-\frac{p}{2}}}{C_{1}(2-p)}$.

(b) For $1<p \leq \frac{2 N}{N+2}$, choose $s=\frac{2 N-(N+1) p}{p}>1$ in (3.1). Again by using the Sobolev inequality and the choice of $s$, one obtains

$$
\|u\|_{s+1}^{\frac{p+s-1}{p}}=\left\|u^{\frac{p+s-1}{p}}\right\|_{\frac{N p}{N-p}} \leq \gamma\left\|\nabla u^{\frac{p+s-1}{p}}\right\|_{p} .
$$

The above inequality together with (3.1) gives us

$$
\frac{1}{s+1} \frac{d}{d t} \int_{\Omega} u^{s+1} d x+\frac{\gamma^{-p} s p^{p}}{(s+p-1)^{p}}\left(\int_{\Omega} u^{s+1} d x\right)^{\frac{p+s-1}{s+1}} \leq a|\Omega|^{\frac{s+2-q}{s+1}}\left(\int_{\Omega} u^{s+1} d x\right)^{\frac{q+s}{s+1}},
$$


or equivalently,

$\frac{1}{s+1} \frac{d}{d t} \int_{\Omega} u^{s+1} d x \leq-\left(\int_{\Omega} u^{s+1} d x\right)^{\frac{p+s-1}{s+1}}\left(\frac{\gamma^{-p} s p^{p}}{(s+p-1)^{p}}-a|\Omega|^{\frac{s+2-q}{s+1}}\left(\int_{\Omega} u^{s+1} d x\right)^{\frac{q+1-p}{s+1}}\right)$.

If $u_{0}(x)$ or $a$ or $|\Omega|$ is small enough such that

$$
\frac{\gamma^{-p} s p^{p}}{(s+p-1)^{p}}>a|\Omega|^{\frac{s+2-q}{s+1}}\left(\int_{\Omega} u_{0}^{s+1} d x\right)^{\frac{q+1-p}{s+1}}
$$

and then we have

$$
\frac{1}{s+1} \frac{d}{d t} \int_{\Omega} u^{s+1} d x \leq-C_{2}\left(\int_{\Omega} u^{s+1} d x\right)^{\frac{p+s-1}{s+1}}
$$

where

$$
C_{2}=\frac{\gamma^{-p} s p^{p}}{(s+p-1)^{p}}-a|\Omega|^{\frac{s+2-q}{s+1}}\left(\int_{\Omega} u_{0}^{s+1} d x\right)^{\frac{q+1-p}{s+1}}>0 .
$$

Integrating $(3.5)$ over $(0, t)$, we have

$$
\int_{\Omega} u^{s+1} d x \leq\left[\left(\int_{\Omega} u_{0}^{s+1} d x\right)^{\frac{2-p}{s+1}}-(2-p) C_{2} t\right]_{+}^{\frac{s+1}{2-p}} .
$$

Thus we see that $u$ vanishes at the finite time $T=\frac{\left(\int_{\Omega} u_{0}^{s+1} d x\right)^{\frac{2-p}{s+1}}}{(2-p) C_{2}}$.

Step 2: $q>1$. Since $q>p-1$, it is easy to verify that $v=k \phi(x)$ is a super-solution of (1.1) for sufficiently small $k>0$ if $u_{0}(x) \leq k \phi(x)$ in $\Omega$, where $\phi(x)$ is the unique positive solution of the following elliptic problem

$$
-\operatorname{div}\left(|\nabla \phi|^{p-2} \nabla \phi\right)=1, x \in \Omega ; \phi(x)=0, x \in \partial \Omega .
$$

Throughout this paper, we denote

$$
M=\sup _{x \in \Omega} \phi(x)>0, \quad \mu=\int_{\Omega} \phi^{p-1}(x) d x>0 .
$$

Then we know by Lemma 2.1 that $u \leq k \phi(x) \leq k M$ for all $(x, t) \in \Omega \times(0, \infty)$. In this case, (3.1) can be rewritten as

$\frac{1}{s+1} \frac{d}{d t} \int_{\Omega} u^{s+1} d x+\frac{s p^{p}}{(s+p-1)^{p}} \int_{\Omega}\left|\nabla u^{\frac{s+p-1}{p}}\right| p d x \leq a k^{q-1} M^{q-1}|\Omega| \int_{\Omega} u^{s+1} d x$.

Then we can apply the above argument to (3.8) to derive the extinction results. The proof of this theorem is complete.

Remark 3.1. In [24], it was proved for the $p$-Laplace equation with a local source that, when $q>p-1$, any bounded and non-negative weak solution vanishes in finite time for appropriately small initial data $u_{0}$. However, one can see from the proof of Theorem 3.1 that the boundedness of the solutions is in fact unnecessary. Moreover, we can see from Theorem 3.1 that small domains can also cause extinctions to occur, which is one of the main differences between the local and nonlocal problems. 
Theorem 3.2. Assume that $0<q<p-1$. Then Problem (1.1) admits at least one nonnegative and non-extinction weak solution $u(x, t)$ for any nonnegative initial datum $u_{0} \in L^{\infty}(\Omega) \cap W_{0}^{1, p}(\Omega)$.

Proof. We shall prove this theorem by using the weak super and sub-solution method. Consider the following problem

$$
\begin{cases}w_{t}=\operatorname{div}\left(|\nabla w|^{p-2} \nabla w\right)+a \int_{\Omega}\left(w_{+}+1\right)^{q}(y, t) d y, & x \in \Omega, \quad t>0 \\ w(x, t)=0, & x \in \partial \Omega, t>0 \\ w(x, 0)=u_{0}(x), & x \in \Omega .\end{cases}
$$

It is obvious that if $w$ is a weak solution of (3.9), then by the weak minimum principle we have $w \geq 0$. By using Schaefer's Fixed Point Theorem, similar to the proof of Proposition 2.1 in [24], we may show that Problem (3.9) admits at least one weak solution. In addition, the weak solution is also a weak supersolution of Problem (1.1). We next construct a suitable weak sub-solution of Problem (1.1) by using the solution of (3.6).

Set $v(x, t)=g(t) \phi(x)$, where $\phi(x)$ is the unique solution of (3.6) and $g(t)$ satisfies

$$
\left\{\begin{array}{l}
g^{\prime}(t)=M^{-1}\left(-g^{p-1}(t)+a \int_{\Omega} \phi^{q}(x) d x \cdot g^{q}(t)\right), \\
g(0)=0, g(t)>0 \text { for } t>0 .
\end{array}\right.
$$

It is not difficult to see that $g(t)$ is a non-decreasing and bounded function, and we can verify directly that $v(x, t)$ is a weak sub-solution of Problem (1.1). Indeed, $v(x, t)$ satisfies the following inequality (in the weak sense)

$$
\begin{aligned}
v_{t} & =g^{\prime}(t) \phi(x) \leq g^{\prime}(t) M=-g^{p-1}(t)+a \int_{\Omega} \phi^{q}(x) d x \cdot g^{q}(t) \\
& =\operatorname{div}\left(|\nabla v|^{p-2} \nabla v\right)+a \int_{\Omega} v^{q}(y, t) d y .
\end{aligned}
$$

Now we have constructed a pair of weak super and sub-solution $w, v$. If $v \leq w$, then the problem admits a weak solution $u$ satisfying $v \leq u \leq w$. Therefore, we need only to show that $v \leq w$.

From the definition of $w, v$, we deduce

$$
\begin{aligned}
& \iint_{Q_{t}} \frac{\partial(v-w)}{\partial t} \varphi(x) d x d \tau+\iint_{Q_{t}}\left(|\nabla v|^{p-2} \nabla v-|\nabla w|^{p-2} \nabla w\right) \nabla \varphi d x d \tau \\
\leq & a \iint_{Q_{t}}\left(\int_{\Omega}\left(v^{q}-\left(w_{+}+1\right)^{q}\right) d y\right) \varphi d x d \tau .
\end{aligned}
$$

Take $\varphi=(v-w)_{+}$where $s_{+}=\max \{s, 0\}$, then we obtain

$$
\begin{aligned}
& \frac{1}{2} \int_{\Omega}(v-w)_{+}^{2} d x+\iint_{Q_{t}}\left(|\nabla v|^{p-2} \nabla v-|\nabla w|^{p-2} \nabla w\right) \nabla(v-w)_{+} d x d \tau \\
\leq & a \iint_{Q_{t}}\left(\int_{\Omega}\left(v^{q}-\left(w_{+}+1\right)^{q}\right) d y\right)(v-w)_{+} d x d \tau
\end{aligned}
$$




$$
\begin{aligned}
& \leq a q \int_{0}^{t}\left(\int_{\Omega}\left(v-\left(w_{+}+1\right)_{+} d x \cdot \int_{\Omega}(v-w)_{+} d x\right)\right) d \tau \\
& \leq a q|\Omega| \iint_{Q_{t}}(v-w)_{+}^{2} d x d \tau .
\end{aligned}
$$

Thus we know by recalling Gronwall's inequality that

$$
\int_{\Omega}(v(x, t)-w(x, t))_{+}^{2} d x=0 \text { for all } t>0
$$

which implies that $v \leq w$ a.e. in $Q$. Hence Problem (1.1) admits a weak solution $u \in\langle v, w\rangle$. Since $v(x, t)$ does not vanish, neither does $u$. The proof is complete.

For the critical case $q=p-1$, whether the solutions to (1.1) vanish in finite time or not depends on the value of $a \mu$, where $\mu$ is defined in (3.7). Precisely, we have the following result in this direction.

Theorem 3.3. Assume that $q=p-1$.

(i) If $a \mu>1$, then Problem (1.1) admits at least one nonnegative and nonextinction weak solution $u(x, t)$ for any nonnegative initial datum $u_{0} \in L^{\infty}(\Omega) \cap$ $W_{0}^{1, p}(\Omega)$

(ii) If a $\mu=1$, then Problem (1.1) admits at least one non-extinction solution $u(x, t)$ for any positive initial datum $u_{0}$;

(iii) If a $<1$, then Problem (1.1) admits at least one solution which vanishes in finite time for any nonnegative initial datum $u_{0}$.

Proof. Case (i). We shall prove this case by using the weak super and subsolution method again. As in the proof of Theorem 3.2 we choose the solution $w(x, t)$ of $(3.9)$ as a weak super-solution of (1.1). We need only to construct a suitable weak sub-solution.

Define $v(x, t)=g(t) \phi(x)$, where $\phi(x)$ is the unique solution of (3.6) and $g(t)$ is a solution of the following ordinary differential equation

$$
\left\{\begin{array}{l}
g^{\prime}(t)=M^{-1}(a \mu-1) g^{p-1}(t) \\
g(0)=0, g(t)>0 \text { for } t>0
\end{array}\right.
$$

Direct computation yields that (in the weak sense)

$$
\begin{aligned}
v_{t} & =g^{\prime}(t) \phi(x) \leq g^{\prime}(t) M=(a \mu-1) g^{p-1}(t) \\
& =\operatorname{div}\left(|\nabla v|^{p-2} \nabla v\right)+a \int_{\Omega} v^{p-1} d x .
\end{aligned}
$$

It is clear that $v(x, t)=0$ for $(x, t) \in \partial \Omega \times(0, \infty) \cup \Omega \times\{t=0\}$. This together with the above inequality shows that $v(x, t)$ is a weak sub-solution of Problem (1.1).

With the weak super-solution and sub-solution in hand, we can prove, by applying the same argument as in Theorem 3.2 , that $v(x, t) \leq w(x, t)$, which implies that Problem (1.1) admits at least one weak solution $u$ satisfying $v \leq$ 
$u \leq w$. Since $v(x, t)$ is positive for all $t>0, u(x, t)$ cannot vanish in any finite time.

Case (ii). Since $q=p-1$ and $a \mu=1$, we see that $v=k \phi$ is a steady state solution of (1.1). Then for any positive initial datum $u_{0}(x)$, we can choose $k>0$ small enough such that $v=k \phi$ is a non-extinction sub-solution of (1.1). Thus we can utilize the method almost the same as in Case (i) to prove the non-extinction result, and the details are omitted.

Case (iii). Set $v(x, t)=g(t) \phi(x)$, where $\phi(x)$ is the unique positive solution of (3.6) and $g(t)$ satisfies

$$
\left\{\begin{array}{l}
g^{\prime}(t)=-\frac{1-a \mu}{M} g^{p-1}(t), t>0 \\
g(0)=A
\end{array}\right.
$$

where $M$ is given in (3.7) and $A>0$ is large enough such that $u_{0}(x) \leq A \phi(x)$ for $x \in \Omega$. Since $0<p-1<1$ and $1-a \mu>0$, we know from the theory of ODEs that $g(t)$ vanishes at a finite time $T_{0}$. On the other hand, it is easily seen that $v(x, t)$ satisfies (in the weak sense)

$$
\begin{aligned}
v_{t}=g^{\prime}(t) \phi(x) & \geq M g^{\prime}(t)=-(1-a \mu) g^{p-1}(t) \\
& =\operatorname{div}\left(|\nabla v|^{p-2} \nabla v\right)+a \int_{\Omega} v^{p-1} d x .
\end{aligned}
$$

Thus, $v(x, t)$ is a weak super-solution of Problem (1.1). It is obvious that 0 is a weak sub-solution of (1.1) and $0 \leq v$. By using the same argument as in the proof of Theorem 3.2 we know that Problem (1.1) admits at least one solution $\widetilde{u}$ satisfying $0 \leq \widetilde{u} \leq v$. Since $v(x, t)$ vanishes at the finite time $T_{0}$, so does $\widetilde{u}$. The proof is complete.

Remark 3.2. Under the hypotheses of Theorem 3.3 Case (iii) we can also estimate the extinction rate of the $L^{r}$ norm of the solution for some $r>1$. This can be done by using almost the same argument as that of Theorem 3.1.

Remark 3.3. It can be seen from Theorems 3.1-3.3 that when $q<p-1$ or $q=p-1$ with $a \mu>1$, Problem (1.1) admits at least one non-negative nonextinction solution, which means that the effect of the source is in some sense strong and the diffusion term can not dominate the source term. However, when $q>p-1$ or $q=p-1$ with $a \mu<1$, which implies that the nonlocal source is a little weak, the effect of the fast diffusion term may cause the nonnegative weak solutions to vanish in finite time, provided that the initial data are sufficiently small. Finally, for the critical case $q=p-1$ with $a \mu=1, k \phi(x)$ with $k>0$ is a steady state solution of (1.1). Then for any positive initial datum, (1.1) admits a solution which is positive in $\Omega$ for all $t>0$.

Acknowledgements. The authors would like to express their deep thanks to the reviewers for their valuable suggestions for the revision and improvement of the manuscript. 


\section{References}

[1] J. G. Berryman and C. J. Holland, Stability of the separable solution for fast diffusion, Arch. Ration. Mech. Anal. 74 (1980), no. 4, 379-388.

[2] E. Dibenedetto, Degenerate Parabolic Equations, Springer, New York, 1993.

[3] R. Ferreira and J. L. Vazquez, Extinction behavior for fast diffusion equations with absorption, Nonlinear Anal. 43 (2001), 943-985.

[4] A. Friedman and Miguel A. Herrero, Extinction properties of semilinear heat equations with strong absorption, J. Math. Anal. Appl. 124 (1987), no. 2, 530-546.

[5] A. Friedman and S. Kamin, The asymptotic behavior of gas in an $n$-dimensional porous medium, Trans. Amer. Math. Soc. 262 (1980), no. 2, 551-563.

[6] J. Furter and M. Crinfeld, Local vs. nonlocal interactions in population dynamics, J. Math. Biol. 27 (1989), no. 1, 65-80.

[7] V. A. Galaktionov and J. R. King, Fast diffusion equation with critical Sobolev exponent in a ball, Nonlinearity 15 (2002), no. 1, 173-188.

[8] V. A. Galaktionov, L. A. Peletier, and J. L. Vazquez, Asymptotics of the fast-diffusion equation with critical exponent, SIAM J. Math. Anal. 31 (2000), no. 5, 1157-1174.

[9] V. A. Galaktionov and J. L. Vazquez, Asymptotic behaviour of nonlinear parabolic equations with critical exponents. A dynamical systems approach, J. Funct. Anal. 100 (1991), no. 2, 435-462.

[10] _ Extinction for a quasilinear heat equation with absorption I. Technique of intersection comparison, Comm. Partial Differential Equations 19 (1994), no. 7-8, 1075-1106.

[11] _ Extinction for a quasilinear heat equation with absorption II. A dynamical system approach, Comm. Partial Differential Equations 19 (1994), no. 7-8, 1107-1137.

[12] Y. G. Gu, Necessary and sufficient conditions of extinction of solution on parabolic equations, Acta. Math. Sinica 37 (1994), 73-79 (in Chinese).

[13] Y. Z. Han and W. J. Gao, Extinction for a fast diffusion equation with a nonlinear nonlocal source, Arch. Math. (Basel) 97 (2011), no. 4, 353-363.

[14] M. A. Herrero and J. J. L. Velazquez, Approaching an extinction point in onedimensional semilinear heat equations with strong absorptions, J. Math. Anal. Appl. 170 (1992), no. 2, 353-381.

[15] C. H. Jin, J. X. Yin, and Y. Y. Ke, Critical extinction and blow-up exponents for fast diffusive polytropic filtration equation with sources, Proc. Edinb. Math. Soc. (2) 52 (2009), no. 2, 419-444.

[16] A. S. Kalashnikov, The nature of the propagation of perturbations in problems of nonlinear heat conduction with absorption, USSR Comp. Math. Math. Phys. 14 (1974), 70-85.

[17] Some problems of the qualitative theory of second-order nonlinear degenerate parabolic equations, Uspekhi Mat. Nauk 42 (1987), no. 2, 135-176.

[18] F. C. Li and C. H. Xie, Global and blow-up solutions to a p-Laplacian equation with nonlocal source, Comput. Math. Appl. 46 (2003), no. 10-11, 1525-1533.

[19] Y. X. Li and J. C. Wu, Extinction for fast diffusion equations with nonlinear sources, Electron J. Differential Equations 2005 (2005), no. 23, 1-7.

[20] W. Liu, Extinction and non-extinction of solutions for a nonlocal reaction-diffusion problem, Electron. J. Qual. Theory Differ. Equ. 2010 (2010), no. 15, 1-12.

[21] W. Liu and $\mathrm{B}$. Wu, A note on extinction for fast diffusive p-Laplacian with source, Math. Methods Appl. Sci. 31 (2008), no. 12, 1383-1386.

[22] E. S. Sabinina, On a class of non-linear degenerate parabolic equations, Dolk. Akad. Nauk SSSR 143 (1962), 794-797.

[23] Y. Tian and C. L. Mu, Extinction and non-extinction for a p-Laplacian equation with nonlinear source, Nonlinear Anal. 69 (2008), no. 8, 2422-2431.

[24] J. X. Yin and C. H. Jin, Critical extinction and blow-up exponents for fast diffusive p-Laplacian with sources, Math. Methods Appl. Sci. 30 (2007), no. 10, 1147-1167. 
[25] J. X. Yin, J. Li, and C. H. Jin, Non-extinction and critical exponent for a polytropic filtration equation, Nonlinear Anal. 71 (2009), no. 1-2, 347-357.

[26] H. J. Yuan, S. Z. Lian, W. J. Gao, X. J. Xu, and C. L. Cao, Extinction and positivity for the evolution p-Laplacian equation in $R^{N}$, Nonlinear Anal. 60 (2005), no. 6, 1085-1091.

YUZHU HAN

Institute of Mathematics

JILIN UNIVERSITY

Changchun 130012, P. R. China

E-mail address: hanyuzhu2003@yahoo.cn

Wenjie GaO

Department of Mathematics

JiLIN UNIVERSITY

Changchun 130012, P. R. China

E-mail address: wjgao@jlu.edu.cn

Haixia Li

Department of Mathematics

JILIN UNIVERSITY

Changchun 130012, P. R. China

E-mail address: lihaixia20080512@126.com 